\title{
Physical Computing Toydesign: Juguetes Interactivos por Medio de Arduino para Niños con Discapacidad Motriz
}

\author{
Physical Computing Toydesign: Interactive Toys made whit Arduino for Children with Motor Disabilities
}

\author{
Bárbara Echaíz Bielitz \\ Universidad de Chile, Chile \\ b.echaiz.b@gmail.com \\ Carla Ponzano Quintanar \\ Universidad de Chile, Chile \\ carlaponzano@gmail.com
}

\begin{abstract}
This research project explores the Arduino potentialities, as a performer and mediator between individuals and their environment. Our goal is to generate spaces to modify that environment through an interface that dialogues with stimuli and actions.Our interest is to connect vulnerable individuals to possible acts of appropriation that is, being able to perform a symbolic ownership of their environments. For doing that, we see the opportunity of using Arduino within the context of differential pedagogy for children with special needs. All this, considering the evident deficiencies of that kind of educational facilities, particularly regarding children with motor disabilities daily activities.
\end{abstract}

Keywords: Physical computing; Arduino; Early education and development; Motor disabilities; Interactive toys.

\section{Introducción}

El objetivo de este documento es proporcionar una guía para Esta investigación se centra en las posibilidades que otorga el Physical Computing para establecer relaciones entre sujeto y entorno, a través de componentes computacionales, mediante flujos informáticos que generan lazos comunicativos entre individuo e interfaz.

El diseño de objetos con estas características presenta componentes elementales para su comunicación. Contando con sensors y actuators que decodifican estímulos del entorno logrando una relación entre lo exterior y el objeto; siendo ésta, mediada por un software alojado en un microcontrolador (Banzi, 2008). Arduino, es la plataforma de microcontroladores más utilizada a nivel mundial, el trabajo con esta placa desarrolla un entorno participativo, creativo y experimental, posibilitando instancias de aprendizaje dentro de un marco artístico-creativo, fomentando habilidades colaborativas y didácticas con un grupo de usuarios.

Esto se transforma en una oportunidad para facilitar las acciones de quienes son más vulnerables de poder realizar cambios en su contexto ambiental. Por esta razón, utilizaremos como foco de estudio a los menores con discapacidad motriz.
Los menores corresponden al primer nivel de enseñanza del Colegio Amapolas, establecimiento de orden municipal ubicado en Ñuñoa, especializado en la Educación Temprana de niños, niñas y jóvenes con trastorno motor.

Dentro de la metodología del colegio los docentes adaptan juguetes que utilizan para la enseñanza, esperando un mejor control por los niños, ya que la accesibilidad a productos especialmente diseñados para este tipo de necesidades es reducida o nula.

Por lo tanto, nuestro objetivo será relacionar la interfaz que propone Arduino con el desarrollo motor de menores que presentan dificultades físicas, extendiendo sus capacidades corporales, donde por medio de acciones didácticas-interactivas, propuestas en un juguete, se estimule la ejercitación de sus deficiencias motoras al mismo tiempo que están aprendiendo, y por sobre todo, recreándose.

Al contar con estos antecedentes, se debe aclarar que la investigación se desarrolla en dos etapas, en primer lugar se encuentra el levantamiento de datos bibliográficos, complementado por entrevistas a especialistas del tema; siendo seguida por una etapa creativa, donde a través de la 
experimentación de prototipos, se busca obtener la mayor cantidad de información para el desarrollo del estudio.

Los objetivos específicos son:

a) Identificar las habilidades básicas de los distintos niveles de trastorno motor para determinar movimientos posibles de realizar en un grupo general.

b) Identificar los tipos de interacción entre estímulos y reacciones que se pueden dar mediante la utilización de Arduino, para la articulación de una interfaz adecuada a la muestra en estudio.

c) Establecer criterios útiles necesarios para el desarrollo de herramientas didácticas que reaccionen mediante estímulos físicos, en base al marco teórico de la Educación Temprana.

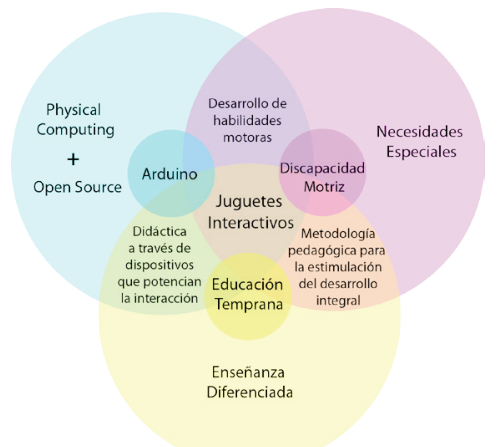

Figura 1: Diagrama de Venn. Temáticas abordadas, elaboración propia

\section{Estrategia Metodológica Enfocada al Usuario y la Exploración Tecnológica}

Metodología creativa: Argumentos del proyecto, espacio para la exploración.

Al ser el Diseño una disciplina creativa, se siente la necesidad de desarrollar la investigación con un carácter experimental y especulativo. Por lo que se explora por medio de constantes iteraciones en la formulación de ideas y en la propuesta de posibles prototipos.

Esta investigación responde a un caso de estudio real, donde hacemos uso, principalmente, de la observación etnográfica como instrumento crucial para los acercamientos más importantes en la experiencia del usuario, sin embargo, el fin de contar con un usuario determinado es usado como base para una exploración creativa en la práctica del producto final. De esta manera, el recorrido metodológico responde en su mayoría a la técnicasandbox, originada en el MIT Media Lab (Koskinen Zimmerman, Binder, Redström, Wensveen, 2011) y utilizada por varias prestigiosas escuelas de diseño, donde encontramos la validación de una exploración tecnológica, mediante constantes iteraciones de prototipado. "Ellos demostraron que es posible hacer investigación con cosas en la mano, sin justificaciones y complejas bases teóricas y dejar que la imaginación suelta en el taller."[6](Koskinen et al., 2011, p.24)
Entonces, es mediante la prospectiva -según Berger (Moles, 1974) que podemos crear el vínculo exploratorio entre lo especulativo y experimental. Donde se origina nuestra metodología de investigación, cuya esencia está en la observación, el prototipado y las constantes repeticiones en el hacer. Cada fase de la metodología tiene una orientación específica, desarrollada mediante la observación etnográfica o en el prototipado con una práctica concreta.

\section{Actividades e instrumentos}

Planteamiento de hipótesis y diseño de dispositivos: Instrumento: propuestas de estímulos-respuesta

Prueba en campo del prototipo-maqueta con menor Instrumento: prototipos a modo de maqueta

Visita a la Teletón:

Instrumento: observación y conversación guiada mediante tópicos.

Entrevista en profundidad a directora del colegio amapolas: instrumento: entrevista en profundidad

Visita colegio amapolas, prueba prototipo:

Instrumento: prueba de prototipo con menores

Las actividades realizadas sirven para definir la aplicación, el concepto y la forma del juguete. Si bien el orden no es el que comúnmente -o tradicionalmente- se desarrolla en un proyecto de Diseño, lo hemos alterado, para seguir lo que las constantes iteraciones y los acercamientos hacia los niños van otorgando.

Datos recopilados

Planteamiento de hipótesis y diseño de dispositivos

Se realizó un brainstormig de acciones posibles que podrían realizar los menores, en relación a la primera visita de acercamiento, así como también un listado de estímulos, que serían gratos para los menores al recibirlos, como:

Acciones

Tacto: Apretar, Rascar, Aplaudir, Aproximar, Acariciar, Mover, Golpear, Abrazar, Flectar, Tapar.

Otros: Gritar, Soplar, Acercarse, Levantar, Ver, Escuchar, Morder, Girar, Pintar, Cubrir.

\section{Estímulos}

Sonidos, Video, Luz, Vibración, Calor, Textura, Música, Voces, Olor, Color, Viento, Imagen.

Por lo que se proponen herramientas didácticas con las que es posible experimentar el actuar de los menores. A modo de acercamiento, se seleccionan cuatro sensores que al activarlos pueden generar un estímulo que el menor sea capaz de percibir, como:

Apretar / Imagen Visual: Mediante las variables que se producen al apretar un sensor de presión, Arduino codificaba la acción hacia una visualización representada en Processing.

Soplar / Luces: Este prototipo utiliza un sensor de vibración como in-put y 8 leds de colores como out-put. Por medio del soplido del 
niño en un cono de cartón y dependiendo de su intensidad prende los leds potencialmente.

Flexión / Sonido: Este prototipo utiliza como interfaz física un flexsensor. Codificando el rango de acción al flectar el sensor, Arduino responderá por medio de un buzzer que sonará dependiendo de la presión ejercida.

\section{Prueba en campo con prototipo-maqueta}

Se le presentaron los prototipos de juguetes en un entorno familiar al menor, de esta manera, se le entregaban una a una las maquetas. La menor se acercaba e interactuaba con los prototipos y sólo en algunas ocasiones hubo que asesorarla en cómo manipularlos. La evaluación se realizó en base a los niveles de atracción, entre la acción ejercida y la respuesta otorgada por el dispositivo. Los datos recogidos de la actividad se clasificaron en:

- Estímulo al ejercer la acción

- Atractivo de la respuesta

- Evolución en la acción (desarrollo paulatino)

- Iteraciones en la acción

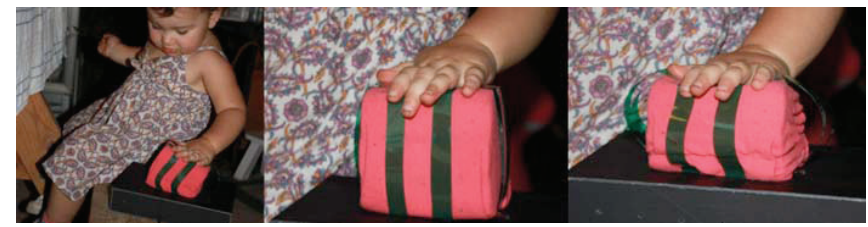

Figura 2: Prueba Maqueta-prototipo. Registro Personal.

De los tres prototipos, el mejor evaluado según el estímulo en ejercer la acción y el atractivo de respuesta que otorgaba el dispositivo fue Flexión/Sonido, ya que la menor volvió en reiteradas ocasiones a jugar con este prototipo. La facilidad en emplear la acción, y la respuesta sonora le hacía interactuar con este juguete.

\section{Visita a Teletón}

Durante la visita a la Teletón guiada por, el encargado del Área Tecnológica, Rodrigo Cubillos, se recorre en detalle la institución, donde fueron especificadas las funciones y particularidades en que se desarrolla cada área de rehabilitación (evolución) y/o ejercitación (práctica-estimulante).

Del universo total de menores con dificultades motrices, algunos van a mejorar sus capacidades y quizás logren hasta un $100 \%$ de evolución y otros, en cambio, evolucionarán en un nivel mínimo o quizás nulo. En estos casos, se ejercita al niño desde sus posibilidades, mientras que aquellos que poseen un nivel de discapacidad severo, se ejercitan desde un nivel que considera las actividades estímulo-sensoriales.

En relación a los tipos de trastornos musculoesqueléticos presentes en los niños que asisten a la Teletón, Rodrigo comenta que la causa "de mayor prevalencia es la parálisis cerebral", donde se conserva movilidad en extremidades superiores. Acotando nuestro campo de acción, enfocándolo en el desarrollo de una continua ejercitación, basándonos en actos autónomos para la reinserción de niños con discapacidad motriz.

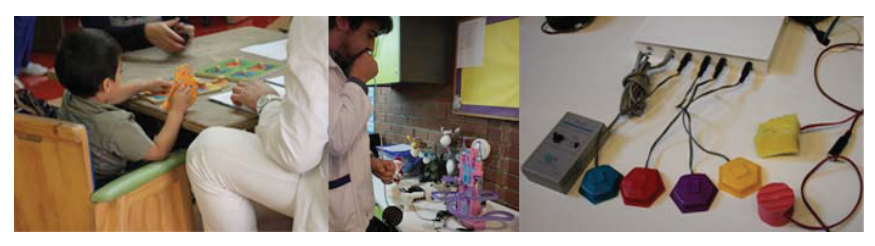

Figura 3: Visita Teletón. Registro Personal

Herramientas de ejercitación para extremidades superiores, utilizadas por Terapeutas Ocupacionales:

Los recursos enfocados a menores discapacitados son limitados, y los habituales no consideran las dificultades que pueden presentar los menores con capacidades restringidas. Principalmente se estimulan habilidades presentes que pueden evolucionar utilizando asociaciones, la ejercitación y el aprendizaje, pero si el juguete no cumple con los requerimientos necesarios, las piezas son adaptadas para posibilitar el uso de cada menor.

\section{Visita a Colegio Amapolas}

Como factor importante en nuestra investigación, se considera a intervenir el nivel educacional más bajo, el Pre-básico (2 años 11 meses a los 7 años 11 meses) donde las clases se dividen en tres núcleos:, ya que los menores estarían susceptibles a recibir mayormente el efecto de la estimulación.

Núcleo Relación con el medio: Grupo humano, Lógico matemático, Seres vivos

Núcleo Comunicación: Lenguaje oral, Lenguaje Escrito, Lenguaje artístico

Núcleo Formación Personal Social: Autonomía, Convivencia, Motricidad y vida saludable

Objetivos del núcleo:

Autonomía: "Adquirir en forma gradual una autonomía que le permita valerse adecuada e integralmente en su medio".

Identidad: "Desarrollar progresivamente una valoración positiva de sí mismo y de los demás, basada en el fortalecimiento de vínculos afectivos con personas significativas que lo aceptan como es."

Convivencia: "Establecer relaciones de confianza, afecto, colaboración, comprensión y pertenencia, basadas en el respeto a las personas y en las normas y valores de la sociedad a la que pertenece"

Recursos didácticos:

Las herramientas que utilizan para la enseñanza son básicas o muy avanzadas a nivel tecnológico, pero en ambos casos no cumplen correctamente con las capacidades físicas de los menores, siendo modificados por los docentes para facilitar su uso. 


\section{Acercamiento a Arduino, inputs y outputs}

Tabla 2 y 3: Elaboración propia de datos del portal en línea adafruit

\begin{tabular}{|c|c|c|}
\hline ENTRADA & USO & INTERACCIÓN \\
\hline \multicolumn{3}{|l|}{ COMPONENTES } \\
\hline Botón & Extremidades & Presión \\
\hline Joystick & Extremidades & $\begin{array}{l}\text { Mov. circular / } \\
\text { empuñadura }\end{array}$ \\
\hline Potenciómetro & Extremidades & $\begin{array}{l}\text { Mov. rotación / } \\
\text { empuñadura }\end{array}$ \\
\hline Micrófono & $\begin{array}{l}\text { Extremidades } \\
\text { / Voz }\end{array}$ & Voz \\
\hline 2.8" Placa táctil TFT & Extremidades & $\begin{array}{l}\text { Mov. superficial- } \\
\text { horizontal }\end{array}$ \\
\hline \multicolumn{3}{|l|}{ SENSORES } \\
\hline Flexión & Extremidades & Flexión \\
\hline Fuerza & Extremidades & Presión \\
\hline Movimiento & $\begin{array}{l}\text { Extremidades / } \\
\text { Cuerpo }\end{array}$ & Movimiento \\
\hline Inclinación & $\begin{array}{l}\text { Extremidades / } \\
\text { Cuerpo }\end{array}$ & Inclinación \\
\hline Luz & $\begin{array}{l}\text { Extremidades / } \\
\text { Cuerpo }\end{array}$ & Aproximación \\
\hline Sonido & Extremidades / Voz & Voz \\
\hline Distancia & $\begin{array}{l}\text { Extremidades / } \\
\text { Cuerpo }\end{array}$ & Aproximación \\
\hline
\end{tabular}

\begin{tabular}{|l|l|}
\hline SALIDA & ESTIMULO / SENTIDOS \\
\hline COMPONENTES & \\
\hline Bomba de aire & "Tacto" \\
\hline Vibrador & "Tacto" \\
\hline Buzzer & Audición \\
\hline Parlante & Audición \\
\hline Led / Leds & Visión \\
\hline Pantalla & Visión \\
\hline $2.8 "$ Placa táctil TFT & Visión \\
\hline
\end{tabular}

Análisis de resultados para las decisiones de Diseño Datos analizados como criterio de conceptualización

Tabla 4: Elaboración propia

\begin{tabular}{|l|l|}
\hline ACTIVIDAD & ANÁLISIS Y CONSIDERACIONES \\
\hline $\begin{array}{l}\text { Prueba de } \\
\text { acercamiento }\end{array}$ & $\begin{array}{l}\text { Considerar el factor estético, y estímulo de } \\
\text { respuesta, como requisitos esenciales en la } \\
\text { fabricación del juguete. Para que sea visualmente } \\
\text { atractivo para el menor. }\end{array}$ \\
\hline Visita a Teletón & $\begin{array}{l}\text { Se rescata el trabajo de los Terapeutas } \\
\text { Ocupacionales, por la satisfacción de los menores } \\
\text { que esperan lograr, a través de la habilidad de } \\
\text { desarrollar actos autónomos. Lo que es posible con } \\
\text { la ejercitación mediante estímulos, acciones y } \\
\text { asociaciones, vinculadas a las herramientas } \\
\text { existentes, las que se centran básicamente en el } \\
\text { agarre de pinza, el reconocimiento de formas y } \\
\text { figuras -encaje- entre otros. }\end{array}$ \\
\hline $\begin{array}{l}\text { Visita a colegio } \\
\text { Amapolas }\end{array}$ & $\begin{array}{l}\text { En el nivel pre-básico, los menores son más } \\
\text { vulnerables a recibir y entender los estímulos del } \\
\text { entorno, lo que incentiva la realización de una } \\
\text { acción que genere una reacción satisfactoria ante } \\
\text { actos voluntarios, por lo que seleccionamos el } \\
\text { Núcleo de Formación Personal Social, que dentro } \\
\text { de sus objetivos consideran la convivencia, donde } \\
\text { estimulan la confianza, el afecto y la comprensión, } \\
\text { basándose tanto en el respeto a las personas como } \\
\text { en las normas y valores de la sociedad. }\end{array}$ \\
\hline
\end{tabular}

\begin{tabular}{|l|l|}
\hline Acercamiento a & Al trabajar con menores que sólo presentan \\
discapacidad motriz, se consideran todos los \\
componentes de salida para crear estímulos, \\
teniendo en cuenta que no pueden ser muchos \\
aplicados en el mismo momento, para facilitar el \\
entendimiento de la acción. En el caso de los \\
componentes de entrada, también hay una amplia \\
consideración, ya que los menores no estarían \\
limitados a manipularlos, sobre todo Arduino \\
permite regular, mediante el código, los rangos \\
establecidos para la reacción de los sensores o \\
componentes.
\end{tabular}

\section{Diseño y construcción de prototipo}

Según lo trabajado en el Núcleo de Formación Personal Social del Nivel Pre-básico, se considera como base el uso de los valores para el desarrollo del menor, lo que fortalece su convivencia y el respeto hacia sus pares. Por lo que se establece como concepto el compartir, que refleja la igualdad de derechos que todos tenemos, y cómo esto influye en nuestros actos.

De esta manera, incluimos las actividades que realizan los terapeutas ocupacionales -que se estima aptas para la mayoría de los menores- como es la empuñadura o agarre en pinza.

\section{Conceptualización y fabricación del prototipo}

Mediante una herramienta didáctica -juguete- se pretende incentivar a los menores a ejercitarse, mediante una narración valórica interactiva. Para generar esto, se disponen en un escenario ficticio de personajes frente a un objeto que deben compartir. Si los protagonistas realizan la acción correcta, es decir, comparten, inmediatamente se genera un estímulo positivo. En cambio, si el objeto no se comparte, por medio de un estímulo se indicará que no se está realizando una buena acción.

En el caso del prototipo, se trabajó con dos ratones y un queso. La intención es poder fabricar según las necesidades y gustos específicos del menor, de esta manera si varían las condiciones ambientales o se quiere agregar más personajes, puede ser modificado el código utilizado, por lo que se debe contar con el software Arduino, descargable desde su portal en línea.
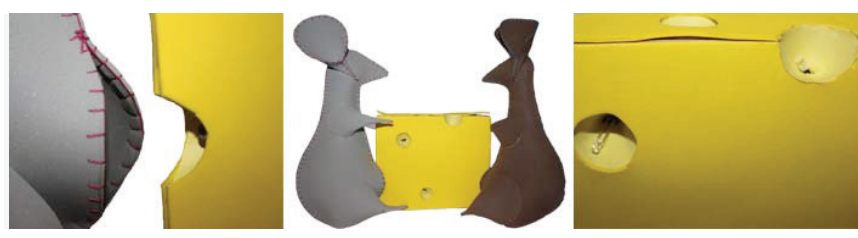

Figura 4: Prototipo Final. Registro Personal

\section{Prueba del prototipo: Interacción de menores con el prototipo}

Se realizó una prueba directa con los menores en la sala de clases, para poder evaluar la experiencia durante la interacción, que involucró tanto a los niños como a los especialistas. La interacción de los menores con el juguete se dio a través de la introducción de un relato que involucra a los personajes, rescatando el valor que se quiere enseñar: compartir. 
Se les muestra lo que ocurre cuando los personajes se mueven, presentándoles lo que se debe realizar para generar ciertos estímulos y reacciones, mediante la articulación de los componentes.

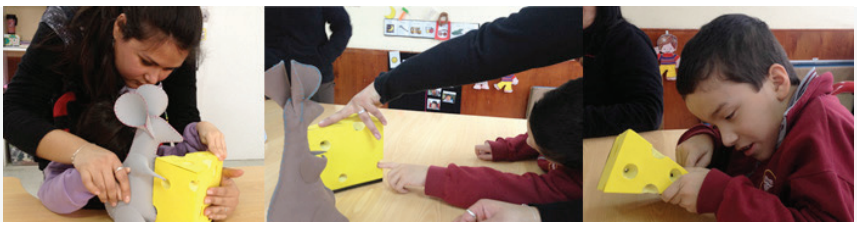

Figura 5: Prueba con Menores con Prototipo Final. Registro Propio

Codigo para Arduino:

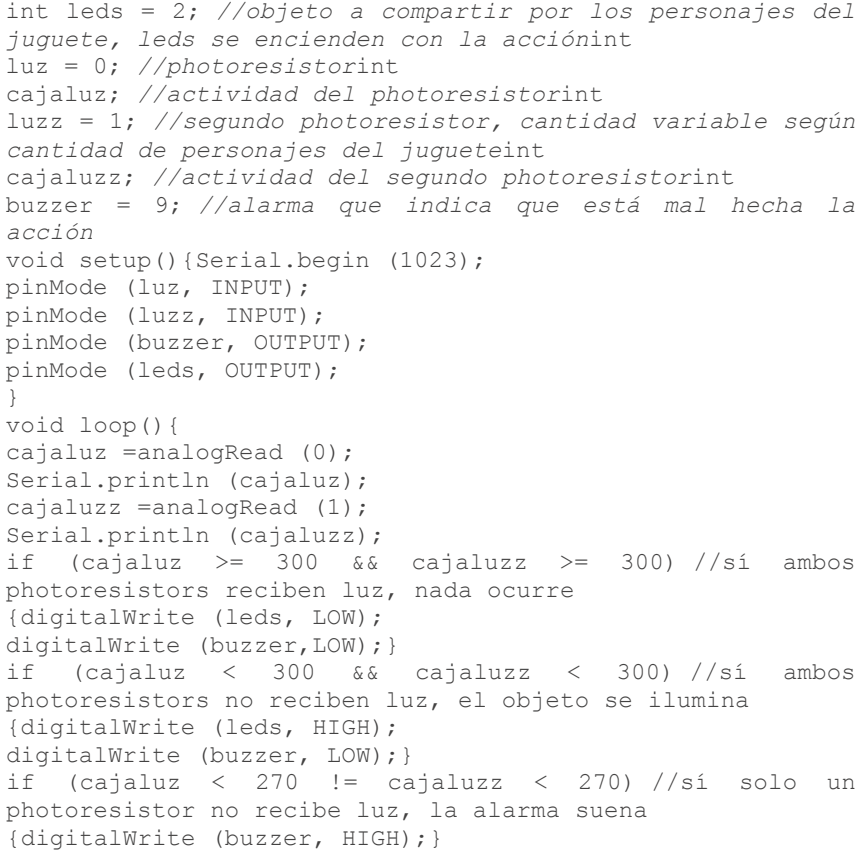

Codigo: Fuente propia

\section{Evaluación según la observación a menores y la opinión de especialistas}

Fue concluyente observar lo que ocurría en durante la prueba, desde el estado anímico de los menores, hasta la relación que se construía a partir del vínculo entre el menor, el juguete y el constante apoyo que tenía el educador en la actividad.

Durante la prueba con los menores, hubo muchas críticas de los educadores, las que manifestaban la necesidad de una ayuda motriz para que la intención del juguete llegará a efecto, sobre todo en cursos como éste que el nivel de actividad motriz entre los niños es muy variada.

\section{Conclusiones}

Era de esperarse que no tuviera un gran porcentaje de éxito la última prueba que realizaron los menores con el prototipo, esto es debido a que es un primer acercamiento, probado y analizado mediante una interacción directa. Si bien tenemos datos que nos proporcionaban una visión un tanto intuitiva para determinar criterios de diseño para un prototipo acabado, no queríamos caer en una definición a priori de lo que es necesario, determinando aquellos criterios con una prueba directa.

Para mejorar los resultados de la interacción con el juguete, se tomará en cuenta la dificultad motriz que presentaron los menores para realizar la acción, esperando facilitarla al incluir guías que restrinjan los movimientos haciendo posible concretar el acto. Siempre considerando que cada menor tiene capacidades diferentes, lo que es muy importante, ya que una de la finalidad de este proyecto es que este juguete sea modificable, es decir, personificable.

\section{Agradecimientos}

Esta investigación fue posible gracias a una inicial experimentación mediante Arduino con Francisco Rojas, Alejandro Pantoja y Diego Gómez. Además, agradecemos de la cordial colaboración del Colegio Amapolas, sobre todo a su directora Bernardita Fontecilla, y al encargado del área tecnológica de la Teletón, Rodrigo Cubillos.

\section{Referencias}

Acevedo, L. (2007). Derecho al Juego, Deporte y Recreación. UNICEF. [versión electrónica] Recuperado 28 de septiembre 2012 de: <http://www.unicef.org/republicadominicana/politics_11167.htm>

Banzi, M. (2008).Getting started with Arduino.Primera ed. O'Reilly.

Ministerio de Educación de Chile. (2007). Guía de apoyo técnicopedagógico: necesidades educativas especiales en el nivel de educación parvularia. Tomo 5: Necesidades educativas especiales asociadas al retraso del desarrollo y discapacidad intelectual. Santiago: MINEDUC. Recuperado el 24 de septiembre 2012, de

<http://www.crececontigo.gob.cl/wp-content/uploads/2009/12/Retrasodel-Desarrollo-y-Discapacidad-Intelectual.pdf/>

Ministerio de Educación de Chile. (2007). Guía de apoyo técnicopedagógico: necesidades educativas especiales en el nivel de educación parvularia. Tomo 3: Necesidades educativas especiales asociadas a discapacidad motora. Santiago: MINEDUC. Recuperado el 24 de septiembre 2012, de

$<$ http://www.crececontigo.gob.cl/wpcontent/uploads/2009/12/Discapacidad-Motora.pdf>

Moggridge, B. (2007). Designing Interactions.Cambridge, MA: MIT Press. 